\title{
Delayed diagnosis of HIV-associated thrombocytopenia in a man of $\mathbf{7 0}$
}

\section{Linda Shavit ${ }^{1} \quad$ Tal Grenader²}

J R Soc Med 2005;98:515

HIV infection is commonly seen as a disease of young people, and failure to consider it in the over-50s can have damaging consequences. In the USA, at least 10\% of AIDS cases now occur in this age group. ${ }^{1}$

\section{CASE HISTORY}

A man of 70, married with three children, was brought to the emergency room after 3 weeks of weakness, dizziness and headache. His only medication was nifedipine for essential hypertension, but for 4 years he had been monitored for thrombocytopenia (platelet counts $70-90 \times 10^{9} / \mathrm{L}$ ). Bone marrow biopsy 3 years earlier had shown hypercellularity with increased red cell precursors and megakaryocytes. Idiopathic thrombocytopenia purpura had been diagnosed, not requiring treatment.

On the present admission the patient's blood pressure was 200/70 mmHg. The Glasgow coma scale was 13 . Neurological examination revealed no localizing signs but he had early papilloedema. Intravenous nitroglycerine was given to control the hypertension. CT showed a large subdural haematoma compressing the right ventricle, with a small amount of fresh haemorrhage. The platelet count was now $5 \times 10^{9} / \mathrm{L}$. Additional investigations for the cause of thrombocytopenia included vitamin B12, folic acid, iron and ferritin, antinuclear antibody, complement $\mathrm{C} 3$ and $\mathrm{C} 4$, and viral serology (Epstein-Barr, cytomegalovirus, hepatitis $\mathrm{B}$ and $\mathrm{C}$ ); all these gave normal results, but the patient was found to have an isolated deficiency of factor VII (15\% of normal). Bone marrow biopsy revealed hypercellularity with a large number of megakaryocytes. The patient was treated with intravenous immunoglobulin (total $1 \mathrm{~g} / \mathrm{kg}$ ) and hydrocortisone and received a platelet transfusion. The platelet count increased transiently to $100 \times 10^{9} / \mathrm{L}$ but fell to $20 \times 10^{9} / \mathrm{L}$ a few days after cessation of immunoglobulin therapy.

${ }^{1}$ Department of Medicine, Sha'are Zedek Medical Center and ${ }^{2}$ Department of Oncology, Hebrew University-Hadassah Medical Center, Jerusalem, Israel

Correspondence to: Tal Grenader, Sharett Institute of Oncology, Hebrew University-Hadassah Medical Center, Jerusalem 91120, Israel

E-mail: talgrenader65@hotmail.com
Three days later a new left-sided hemiparesis developed. Repeat CT showed no enlargement of his subdural haematoma or fresh bleeding. Urgent splenectomy to control the thrombocytopenia was followed by craniotomy and drainage of the subdural haematoma. Postoperatively the platelet count rose to normal $\left(250 \times 10^{9} / \mathrm{L}\right)$ and the neurological signs disappeared. At discharge we received the patient's ELISA result which was positive for HIV infection, confirmed by Western blot analysis. The viral load was high (980 000 copies/mL) and the CD4 count low $(250 / \mu \mathrm{L})$. Antiretroviral therapy (zidovudine, lamivudine, nevirapine) was started, and a year later the patient was free from thrombocytopenia or AIDS-defining illness.

\section{COMMENT}

Although thrombocytopenia is a common and important haematological manifestation of HIV infection, ${ }^{2}$ the advanced age of our patient made this diagnosis initially seem unlikely. The patient was treated with high-dose steroids and immunoglobulins and had two major surgical procedures before the correct diagnosis was made. There has been a previous case report of pancytopenia as the presenting feature of HIV infection in advanced age by Boudes et al. ${ }^{3}$ but thrombocytopenia as the initial manifestation is very unusual. Such thromboctyopenia may manifest clinically with petechiae, ecchymoses, epistaxis, and bleeding from the gums and gastrointestinal tract, but intracranial bleeding is rare, even when the platelet count is very low; in the present case the factor VII deficiency probably contributed.

There is a misconception on the part of both patients and doctors that the elderly are not at risk for HIV-infection. Before this diagnosis is thought of they have often been investigated and treated for conditions such as cerebrovascular disease, Alzheimer's disease, bacterial pneumonia, malnutrition or occult malignancy. ${ }^{4}$ Median lifespan is 9 months compared with 22 months in the young. ${ }^{5}$

Acknowledgments We are grateful to Dr M Lifschitz for his assistance in preparing this report.

\section{REFERENCES}

1 Ship J, Wolff A, Selik R. Epidemiology of acquired immune deficiency syndrome in persons aged 50 years or older. J Aquir Immune Defic Syndr $1991 ; 4: 84-8$

2 Scaradavou A. HIV-related thrombocytopenia. Blood Rev 2002;16:73-6

3 Boudes P, Balloul E, Sobel A. Pancytopenia as the presenting manifestation of HIV infection in the elderly. $J$ Am Geriatr Soc 1989;37:1151-2

4 Sabin TD. AIDS: the new 'great imitator'. J Am Geriatr Soc 1987;36:460-4

5 Chen HX, Ryan PA, Ferguson RP, Yataco A, Markowitz JA, Raksis K. Characteristics of acquired immunodeficiency syndrome in older adults. J Am Geriatr Soc 1998;46:153-6 